$66 \%, 95 \%$, and $64 \%$ of cases respectively. Level of consciousness, motor activity, muscle tone, pupil size and reaction to light were similarly inadequately documented.

This quality improvement initiative aimed to educate doctors in a tertiary neonatal unit regarding the importance of systematic evaluation and documentation of neonatal encephalopathy using the Modified Sarnat Score.

Methods An education session for all medical staff working in the neonatal unit was held, outlining the importance of appropriate use and documentation of the Sarnat Classification in clinical practice. Index cards were issued to all staff, describing the individual components of the score and those techniques necessary to elicit the relevant neurologic signs. A teaching video, demonstrating neurological assessment of a neonate using the Modified Sarnat Score, was recorded and uploaded to the official hospital website. Anonymous questionnaires were obtained to assess effectiveness of teaching.

Results In total, 17 Healthcare Professionals attended the education session, with a mean of 8 years experience working in Paediatrics. 58\% $(n=10)$ had previously received education on the Sarnat Score. $47 \%(n=8)$ had used the Sarnat Score previously in clinical practice. Lack of formal training on the score was the main reason cited amongst those who had not used the score previously. $23 \%(n=4)$ correctly identified the individual components of the score prior to education. $12 \%$ $(\mathrm{n}=2)$ correctly scored $\geq 80 \%$ of the clinical scenarios provided. Following education, $71 \% \quad(n=12)$ correctly scored $\geq 80 \%$ of the clinical scenarios provided. All staff $(n=17)$ found the education session informative, and felt more confident using the Sarnat Score following the session. 58\% $(n=10)$ felt that further education on the Sarnat Score was required.

Conclusion It is recognised that the neurological assessment of infants is challenging, particularly for inexperienced clinicians. The validated Sarnat classification system is a valuable resource in the clinical assessment of neonates with encephalopathy. This clinical tool provides physicians with a standardised approach to systematic neurological examination and documentation of pertinent neurological findings.

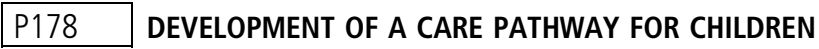 WITH EDWARD'S SYNDROME}

${ }^{1}$ Fiona Finlay, ${ }^{2}$ Richard Lee-Ke*. Virgin Care, Bath, UK; ${ }^{2}$ Bristol Royal Hospital for children, Bristol, UK

10.1136/archdischild-2019-epa.533

Edward's syndrome (Trisomy 18) occurs in approximately 1 in 10,000 live births and is characterised by low birthweight, micrognathia, low-set ears, occipital prominence, clenched fists with overlapping fingers, rocker-bottom feet, cardiac and renal anomalies and developmental delay.

In the past Edward's syndrome has been regarded as a lethal condition, with little intervention other than comfort care being offered following delivery. However, It has become clear that with medical intervention the prognosis for children with Edward's syndrome is better than previously thought, with $5-10 \%$ surviving beyond their first birthday.

Paediatricians have the challenge and the opportunity of helping families face an uncertain future and should engage collaboratively with them to focus on positive life-goal management plans, hopes and aspirations. However some doctors may feel unprepared to manage children these children and have been criticised by parents who say they have focused too much on end-of-life plans, not giving their child a fair chance and creating 'self-fulfilling prophecies' limiting a child's chances.

Along with professionals and parents we have developed guidelines to assist health professionals, giving them a framework for the management of children with Edward's syndrome to try to ensure high quality, evidenced based practice for each individual child. The guideline includes management following diagnosis (antenatal or postnatal); care of mother and baby following delivery; discharge planning; follow up in the community; care in the first weeks, months and year of life; and care of the older child.

The guideline, which will be presented, aims to aid both paediatricians and parents. Ideally parents should feel that as their child grows and develops an individualised care plan is in place and they are clear as to what is going to happen in the future in order to support both them and their child.

\section{P179 PROMOTING AWARENESS OF CLEFT CARE: IMPROVING COLLABORATION \& COMMUNICATION}

Jane Gunn*. Nurse Specialist with the Cleft Team at Temple Street Children's University Hospital, Dublin, Ireland

\subsection{6/archdischild-2019-epa.534}

Background Cleft lip and/or palate affects approximately 1 in 700 live births worldwide. 50\% of the Irish cohort present as a cleft of the palate alone. Up to a third are not detected within the first 24 hours of life. Averages of $60 \%$ with lip involvement are diagnosed on antenatal scanning. Cleft Teams accept referrals upon diagnosis, collaborating and communicating with local services on a Hub \& Spoke model to provide care. Delays in diagnosis and referral times can have a negative impact on the child and family. Some 400 syndromes are associated with clefting, most within the rare diseases category, and may not be diagnosed until months or years after birth.

Aims

- Promote awareness of how cleft care is organized in Ireland.

- Encourage enhanced collaboration \& communication with local hospital and community based services to improve diagnosis rates and referral times.

- Highlight the need for on-going monitoring by key health care professionals (HCPs).

\section{Methods}

- Audits of timing of diagnosis demonstrated.

- Interventions planned by the Cleft Clinical Nurse Specialist (CNSp) detailed.

- Areas for improved working links are discussed.

Results On-going audit demonstrates upward trends in diagnosis rates $-82 \%$ diagnosis in the $1^{\text {st }} 24$ hours of life for 2018 birth cohort with cleft palate only. Education sessions provided by the CNSp to HCPs have received positive feedback. Liaison with Public Health Nurses is proving successful for weight monitoring and cleft surgery planning.

Discussion Although cleft is a specialist area of practice it affects on average 80 families a year in the Republic of Ireland. The Cleft CNSp has a role to educate and collaborate 
with maternity \& community HCPs in highlighting the importance of prompt diagnosis and subsequent referral and management.

Conclusion On-going audit is important to identify trends/ improvements. Continued education sessions for HCPs can assist with increasing awareness of cleft conditions, and the idea of 'link nurses' or 'cleft champions' could be considered. With a changing Model for Children's Healthcare in Ireland further work is required with Neonatology, General Paediatrics, General Practitioners and Genetic teams to formulate more robust pathways for equal access to care and to assist potential syndrome diagnosis. In the interim the Cleft Team propose that each child has access to a named paediatrician locally for $<5$ years of age and that there is enhanced communication between HCPs involved in a child's care.

\section{P180 QUALITY IMPROVEMENT PROJECT OF DISCHARGE SUMMARIES IN OUR LADY OF LOURDES IN DROGHEDA}

${ }^{1}$ Ann-Marie Hayes*, ${ }^{2}$ John Fitzsimmons. 'Our Lady of Lourdes Hospital, Drogheda, Ireland; ${ }^{2}$ Temple Street Children's University Hospital, Dublin, Ireland

\subsection{6/archdischild-2019-epa.535}

Aims Quality improvement (QI) as a concept for clinical research has become more prevalent in recent years. Quality improvement is a focus on changing the way in which patient care is delivered. Central to this is developing a more rounded, patient-focused approach to the design and delivery of care. ${ }^{1}$ Discharge summaries are an important aspect of patient care and having the correct content is essential to communicate treatment plans with general practitioners.

Methods An regular review of discharge summaries on the paediatric ward was conducted in Our Lady of Lourdes Drogheda over a 4 week period. An initial 2 week review of charts was conducted and then an education session was carried out with NCHDs and results were explained. Five areas were considered for analysis, time to discharge summary completion (T2DSC), use of abbreviations (UOA), correct diagnosis documented (CD), correct follow up and referral (FU\&R) and overall impression of discharge summary(OI). A sample of 20 random charts was used. The review was carried out again at two further points after a further education session to assess if there was an improvement.

Results The average for T2DC was $2.7 / 5$ for week $1 \& 2$. It was $3.35 / 5$ for week 4 . UOA was $3.5 / 5$ initially and $3.65 / 5$ after week 4. CD was $3.12 / 4$ and $3.9 / 4$ after week 4. FU\&R was $2.07 / 4$ and $3.8 / 4$ for week 4 . OA was $3 / 5$ and $3.9 / 5$ for week 4.

$\mathrm{P}$ value was 0.64 comparing totals of week $1 \& 3$. Comparing week $1 \& 4 \mathrm{p}$ value was $<0.05$ for all parameters and 0.000003 for total.

Conclusion QI in discharge summaries demonstrated a significant improvement in quality of discharge summaries in OLOL in Drogheda over the time period identified. It demonstrates how QI is a useful tool to improve aspects of clinical practice.

\section{P181 \\ AN AUDIT OF PAEDIATRIC STAFF ATTENDANCE AT PREMATURE DELIVERIES $\leq 32$ WEEKS GESTATION IN CAVAN GENERAL HOSPITAL A LOCAL UNIT}

Seamus Mac Farland ${ }^{*}$, Nick van der Spek. Paediatric Department, Cavan General Hospital., Cavan, Ireland

\subsection{6/archdischild-2019-epa.536}

Background and aims Based on the model of care for neonatal services in Ireland 2015, Cavan General Hospital is classified as a level 1 local unit. Therefore it offers routine and special care to infants $\geq 32$ weeks gestation. Infants $\leq 32$ weeks should usually be transferred to a regional/tertiary unit, preferably in-utero, however this is not always possible. Currently there is no national/local guideline in place to indicate which paediatric staff should be called routinely to attend premature deliveries. The aim of this audit was to establish who currently attends premature deliveries. Also to explore what would be best-practice in a level 1 unit like Cavan and make appropriate recommendations.

Methods A retrospective chart review was carried out of all premature deliveries $\leq 32$ weeks gestation over a 3 year period from 2012 to 2014. Data such as mode of delivery, indication for delivery, members of the resusitation team in attendance and use of steroids were recorded and analysed.

Results In total, 27 deliveries were reviewed, 12 vaginal deliveries and 15 Emergency Cesarean Sections. Based on gestation; < 26/40: $(n=3), 26-30 / 40: \quad(n=7), 30-32 / 40$ : $(n=17)$.

\begin{tabular}{ll}
$\begin{array}{l}\text { Abstract P181 Table } 1 \\
\text { present at } \leq 32 / 40 \text { weeks gestation deliveries }\end{array}$ \\
\hline All in Attendance (Consultant, Reg, SHO, SCBU Nurse) & 3 \\
\hline Reg \& SCBU Nurse & 8 \\
Consultant,Reg, SCBU Nurse & 4 \\
Reg Alone & 4 \\
Consultant, Reg, SHO & 3 \\
Reg, SHO, SCBU Nurse & 2 \\
Consultant \& Reg & 1 \\
Reg \& SHO & 1 \\
Consultant Alone & 1 \\
\hline
\end{tabular}

The Paediatric Registrar was present at 26/27 (96\%) of deliveries, a SCBU Nurse at $18 / 27$ (66.6\%), the Paediatric Consultant at $12 / 27(44 \%)$ and the Paediatric SHO only documented at $9 / 27$ (33.3\%). Of note a paediatric consultant attended all 3 deliveries $<26 / 40$ and $6 / 7$ deliveries between 26-30/40.

Conclusion In our small cohort, there was wide variation with noconsistent practice. Resusitation documentation needs improving. A SCBU Nurse and Paediatric Registrar were the commonest resuscitation team. Deciding on a gestational cut off and defining high-risk deliveries would be helpful in establishing a standardised protocol for when a consultant paediatrician should be in attendance at a delivery. 